\title{
Monitorização Ambulatorial da Pressão Arterial em Indivíduos com Resposta Exagerada dos Níveis Pressóricos em Esforço. Influência do Condicionamento Físico
}

\author{
Eliudem G alvão Lima, Fernando Herkenhoff, Elisardo Corral Vasquez \\ Vitória, ES
}

Objetivo - Avaliar em indivíduos hiper-reatores ao teste ergométrico (TE), a influência de um programa regular de exercícios sobre os parâmetros ergométricos e da $M A P A$.

Métodos - Estudaram-se 22 indivíduos (44 1 anos), sedentários, assintomáticos, normotensos e que apresentavam elevação exagerada da pressão sistólica (PAS $>220 \mathrm{mmHg}$ ) durante o TE, divididos, através de amostragem casual simples, em dois grupos: grupo hiper-reator sedentário (GHS) e grupo hiper-reator condicionado (GHC). Os indivíduos do GHS foram orientados a não realizar qualquer tipo de exercício físico regular durante o período de 4 meses e o GHC composto de 10 indivíduos submetidos a programa de condicionamento físico aeróbico durante o mesmo período.

Resultados - Um programa de exercícios aeróbicos de moderada intensidade não promove redução significativa dos níveis pressóricos durante a monitorização $(P>0,05)$; mas, durante a realização do TE nesses indivíduos, verificamos redução $(p<0,05)$ dos níveis sistólicos e da freqüência cardíaca em cargas submáximas de trabalho.

Conclusão - Um programa de condicionamento aeróbico em hiper-reatores não apresentou alterações significantes nos valores de PA de 24 h e nos sub-periodos; entretanto, durante o TE, observamos redução da atividade inotrópica e cronotrópica em cargas sub-máximas de trabalho, sugerindo o envolvimento do sistema nervoso simpático nas respostas pressóricas exageradas observadas nesses indivíduos durante o exercício dinâmico, e uma redução correspondente do tônus simpático após o treinamento. Este efeito, aparentemente, não se estendeu às suas atividades diárias.

Palavras-chave: teste ergométrico, hipertensão reativa, MAPA.

\section{Ambulatory Blood Pressure Monitoring in Individuals with Exaggerated Blood Pres- sure Response to Exercise, Influence of Exercise Training}

Purpose - To evaluate the effects of exercise training on ergometric test and Ambulatory Blood Pressure Monitoring $(A B P M)$ in normotensive individuals with exaggerated blood pressure response to exercise.

Methods - We studied 22 sedentary and normotensive subjects (mean 44 1 years old) showing an exaggerated systolic blood pressure response ( $\mathrm{SBP}>220 \mathrm{mmHg}) \mathrm{dur}$ ing a cycloergometric test. These individuals were divided in two groups: sedentary hyperreactive group (SHG) and trained hyperreactive group (THG). The THG was submitted to a 4 month aerobic exercise training program.

Results - A program of moderate aerobic exercise did not reduce (P>0.05) ABPM blood pressure levels. However, in the submaximal loads of dynamic exercise we observed a significant fall in the SBP and heart rate $(P<0.05)$.

Conclusion - Normotensive individuals with exaggerated blood pressure response to dynamical exercise submitted to physical training presented a reduction in their systolic blood pressure and heart rate response during the submaximal loads of the cycloergometric test. The ABPM blood pressure values, however, did not change in both groups. These results suggest the involvement of the sympathetic nervous system in the exaggerated blood pressure response observed in these individuals during dynamic exercise and a corresponding reduction of the sympathetic tonus after training. This effect, apparently, did not extend to their daily activities.

Key-words: ABPM, hyperreactive response, ergometric test

Arq Bras Cardiol, volume 70 (n 4), 243-249, 1998

Clínica de Investigação Cardiovascular - CBM, Laboratório de Fisiologia do Exercício - CEFD, UFES - Vitória

Correspondência: Eliudem Galvão Lima - Av. Marechal Campos, 1468 - 29040090 - Vitória, ES

Recebido para publicação em 24/11/97

Aceito em 11/2/98
Sabe-se que o estilo de vida sedentário é fator de risco para o desenvolvimento da hipertensão arterial sistêmica (HAS) e que os exercícios aeróbicos são utilizados como meio terapêutico isolado ou associado ao tratamento farmacológico, visando a redução dos níveis tensionais em indivíduos hipertensos 1 . 
Pesquisas realizadas em animais e em seres humanos sugerem um efeito anti-hipertensivo ao exercício e, assim, o condicionamento físico aeróbico é proposto como forma não farmacológica para o tratamento da $\mathrm{HA}^{2}$. Críticas a esses trabalhos, no que concerne à não utilização de grupo controle, inespecificação dos critérios de randomização, determinação não cega da pressão arterial (PA) e possibilidade de co-intervenção, fazem com que a aceitação da sua eficácia contraponha-se a uma posição mais cautelosa expressa por pesquisadores desta área ${ }^{3}$.

O efeito hipotensor e cardioprotetor da atividade física poderia ser atribuído, diretamente, a mecanismos hemodinâmicos ou, indiretamente, através de modificações nutricionais, metabólicas ou comportamentais. Entre as alterações hemodinâmicas, podem ser citadas a diminuição do débito cardíaco de repouso, do tônus simpático cardiovascular e da resistência vascular periférica ${ }^{4,5,6}$. Entre os fatores nutricionais merecem destaque, a perda de peso, a menor ingestão alcoólica, o aumento da fração HDLcolesterol, a redução do colesterol total e triglicérides, a redução da resistência à insulina ${ }^{7}$. Variações humorais e comportamentais, como: redução dos níveis plasmáticos de catecolaminas, de ouabaina endógena e de volume corpuscular médio do eritrócito, elevação dos níveis de dopamina, prostaglandinas Ee taurina, também, são atribuídas ao exercício. Em adição, a redução de estresse e de ansiedade secundárias ao exercício físico contribuem para diminuir a $\mathrm{PA}^{8}$.

Considerando que a redução dos níveis tensionais em repouso e em esforço tem sido objetivo de programas de atividades aeróbicas regulares em indivíduos sedentários e hipertensos e que na maioria dos estudos, os registros dos efeitos do exercício sobre a PA foram realizados através de esfigmomanômetro durante curtos períodos diários e em condições laboratoriais, objetivamos determinar o efeito de um programa de exercícios aeróbicos realizados em hiperreatores ao exercício dinâmico sobre os níveis tensionais durante as atividades diárias e à noite. Os indivíduos foram submetidos ao teste ergométrico (TE) e à monitorização ambulatorial da pressão arterial (MAPA), a fim de analisarmos as alterações decorrentes do exercício físico sobre os parâmetros estudados. Considerando também que os estudos realizados pela MAPA em normotensos e hipertensos submetidos ao programa de exercícios aeróbicos são controversos, e que não existe trabalho na literatura que faça referência ao condicionamento físico em indivíduos com elevação exagerada da pressão arterial sistólica (PAS) durante o exercício físico, resolvemos estudar este grupo.

\section{Métodos}

A amostra foi constituída de 22 indivíduos do sexo masculino, sedentários, assintomáticos, normotensos em repouso (PAS $\leqslant 140 \mathrm{mmHg}$ e pressão arterial diastólica $(\mathrm{PAD}) \leqslant 90 \mathrm{mmHg}$ ) e com características antropométricas semelhantes (tab. I). Todos funcionários de uma companhia estatal localizada no município de Vitória (ES) e ocupantes de atividades físicas consideradas leves, segundo estimativa de metabolismo adotada pela legislação brasileira9.

\begin{tabular}{|c|c|c|}
\hline Parâmetros & GHS & GHC \\
\hline $\mathrm{n}$ & 12 & 10 \\
\hline Idade (anos) & $45 \pm 1,0$ & $43 \pm 0,9$ \\
\hline Peso $(\mathrm{kg})$ & $78 \pm 2,8$ & $79 \pm 2,9$ \\
\hline Altura & $174 \pm 2,0$ & $173 \pm 2,0$ \\
\hline Índice de Quetelet & $25,5 \pm 0,5$ & $26,2 \pm 0,7$ \\
\hline
\end{tabular}

Após avaliação clínica inicial, os indivíduos foram submetidos ao TE em cicloergômetro, realizado em condições idênticas, com registro eletrocardiográfico contínuo através de um registrador de três canais. Todos os pacientes alcançaram no mínimo $85 \%$ da frequiência cardíaca (FC) prevista para a idade.

A PA durante o TE foi medida através do método indireto convencional, utilizando aparelho tipo aneróide, devidamente calibrado, contra um manômetro de mercúrio. $\mathrm{O}$ ponto de corte para determinação da resposta hiperreativa foi o aumento da PAS > 220 $\mathrm{mmHg}$ durante o esforço ergométrico, considerando como cifras normais em esforço máximoentre $160 \mathrm{mmHg} 2220 \mathrm{mmHg}^{10}$.

Durante a realização dos exames ergométricos, estes indivíduos apresentaram durante o esforço, elevação exagerada dos níveis pressóricos (resposta hiper-reativa), sendo selecionados e constituindo o grupo hiper-reator $(\mathrm{GH}) \mathrm{e}$ dividido em dois grupos: o grupo sedentário (GHS) e o grupo condicionado (GHC). O GHS constituiu-se de 12 indivíduos hiper-reatores que permaneceram durante quatro me-

\begin{tabular}{|c|c|c|c|c|}
\hline \multicolumn{5}{|c|}{$\begin{array}{c}\text { Tabela II - Valores de pressão arterial e de freqüência cardíaca obtidos } \\
\text { em repouso antes (teste 1) e após (teste } 2 \text { ) o programa de condiciona- } \\
\text { mento físico do grupo hiper-reator sedentário (GHS) e grupo hiper- } \\
\text { reator condicionado (GHC) }\end{array}$} \\
\hline \multicolumn{3}{|c|}{ GHS } & \multicolumn{2}{|l|}{ GHC } \\
\hline Parâmetros & teste 1 & teste 2 & teste 1 & teste 2 \\
\hline $\mathrm{n}$ & 12 & 12 & 10 & 10 \\
\hline PAS & $134 \pm 2$ & $133 \pm 2$ & $133 \pm 2$ & $129 \pm 3$ \\
\hline PAD & $83 \pm 1$ & $83 \pm 1$ & $85 \pm 1$ & $83 \pm 2$ \\
\hline FC & $69 \pm 2$ & $72 \pm 3$ & $73 \pm 2$ & $70 \pm 3$ \\
\hline
\end{tabular}

\begin{tabular}{|c|c|c|c|c|}
\hline \multicolumn{5}{|c|}{$\begin{array}{l}\text { Tabela III - Parâmetros ergométricos obtidos antes (teste 1) e após (teste } \\
\text { 2) o programa de condicionamento físico do grupo hiper-reator } \\
\text { sedentário (GHS) e do grupo hiper-reator condicionado (GHC) }\end{array}$} \\
\hline & \multicolumn{2}{|c|}{ GHS } & \multicolumn{2}{|c|}{ GHC } \\
\hline Parâmetros & teste 1 & teste 2 & teste 1 & teste 2 \\
\hline $\mathrm{n}$ & 12 & 12 & 10 & 10 \\
\hline Duração do teste (s) & $738 \pm 30$ & $756 \pm 30$ & $666 \pm 24$ & $756 \pm 36^{*}$ \\
\hline Trabalho total (watts/min) & $1067 \pm 88$ & $1026 \pm 118$ & $964 \pm 47$ & $1155 \pm 84$ \\
\hline
\end{tabular}




\begin{tabular}{|c|c|c|}
\hline \multicolumn{3}{|c|}{$\begin{array}{l}\text { Tabela IV - Valores de duplo produto obtido antes, durante e após o } \\
\text { teste ergométrico no grupo hiper-reator condicionado (n=10) antes } \\
\text { (teste 1) e após (teste 2) o programa de condicionamento físico }\end{array}$} \\
\hline Parâmetros & teste 1 & teste 2 \\
\hline Controle & $9.731 \pm 384$ & $9.147 \pm 549$ \\
\hline Carga 50W & $20.159 \pm 701$ & $16.452 \pm 901 * *$ \\
\hline Carga $75 W$ & $28.017 \pm 976$ & $23.479 \pm 1.454 *$ \\
\hline Carga 100W & $35.809 \pm 1.584$ & $29.656 \pm 1.575 *$ \\
\hline Carga $125 \mathrm{~W}$ & $41.814 \pm 703$ & $38.914 \pm 807 *$ \\
\hline Recuperação 2', & $19.507 \pm 589$ & $16.488 \pm 580 * *$ \\
\hline Recuperação 6', & $15.621 \pm 524$ & $13.596 \pm 538^{*}$ \\
\hline \multicolumn{3}{|c|}{$\begin{array}{l}\text { Valores expressos como média } \pm \text { EPM; duplo produto }=\text { pressão arterial } \\
\text { sistólica' freqüência cardíaca; }{ }^{*}<<0,05 \mathrm{e}^{* *} \mathrm{p}<0,01 \text { (teste } 1 \text { vs teste } 2 \text { ) - teste } \\
\text { t Student. }\end{array}$} \\
\hline
\end{tabular}

ses com suas atividades habituais e sob supervisão médica, sendo orientado a não realizar qualquer tipo de exercício físico durante o período. O GHC constou de 10 indivíduos que foram submetidos a programa de exercícios físicos aeróbicos durante o mesmo período, com prescrição baseada nas orientações do Colégio Americano de Medicina Desportiva ${ }^{11}$.

Após realização do TE, os pacientes foram submetidos à MAPA, através de monitor SpaceLabs modelo 90207. Este aparelho utiliza-se de técnica oscilométrica para mensurações da PA e permite gravação automática/manual da PA e FC durante as $24 \mathrm{~h}$. O monitor era calibrado semanalmente contra um esfigmomanômetro de coluna de mercúrio.

No período de 24h o aparelho foi programado para obter as medidas de $15 \mathrm{em} 15 \mathrm{~min}$ no período de 6:00 às 22:00he de 60em 60min das 22:00 às 6:00h da manhã do dia seguinte. O exame era considerado eficaz quando $80 \%$ das leituras realizadas eram válidas.

Os indivíduos foram instruídos a manterem as suas atividades diárias habituais durante o período de leituras e orientados a permanecer desde que possível, com o membro superior não dominante, contendo o manguito em posição adequada durante cada medida. Foram dadas as devidas instruções para preenchimento detalhado do diário, permitindo correlações com os dados a serem analisados.

O relatório forneceu-nos valores e médias da PA e da FC e análises gráficas do comportamento desses parâmetros durante as $24 \mathrm{~h}$. As normas para interpretação dos resultados foram as definidas pelo Consenso Brasileiro de Monitorização Ambulatorial da Pressão Arterial ${ }^{12}$.

\begin{tabular}{|lcc|}
\hline \multicolumn{2}{|c|}{$\begin{array}{c}\text { Tabela V - Valores de duplo produto obtido antes, durante e após o teste } \\
\text { ergométrico no grupo hiper-reator sedentário (n=12) } \\
\text { aptes (teste 1) e } \\
\text { após (teste 2) período de sedentarismo. }\end{array}$} \\
\hline Parâmetros & teste 1 & teste 2 \\
\hline Controle & $9.264 \pm 438$ & $9.696 \pm 547$ \\
Carga 50W & $17.553 \pm 1.035$ & $17.825 \pm 903$ \\
Carga 75W & $24.139 \pm 1.121$ & $23.641 \pm 998$ \\
Carga 100W & $30.742 \pm 1.445$ & $29.740 \pm 1.353$ \\
Carga 125W & $38.790 \pm 778$ & $29.740 \pm 1.353$ \\
Recuperação 2' & $15.803 \pm 689$ & $16.902 \pm 877$ \\
Recuperação 6' & $13.364 \pm 557$ & $13.675 \pm 648$ \\
\hline Valores expressos como média \pm EPM; duplo produto = pressão arterial sistólica \\
frequiência cardíaca.
\end{tabular}

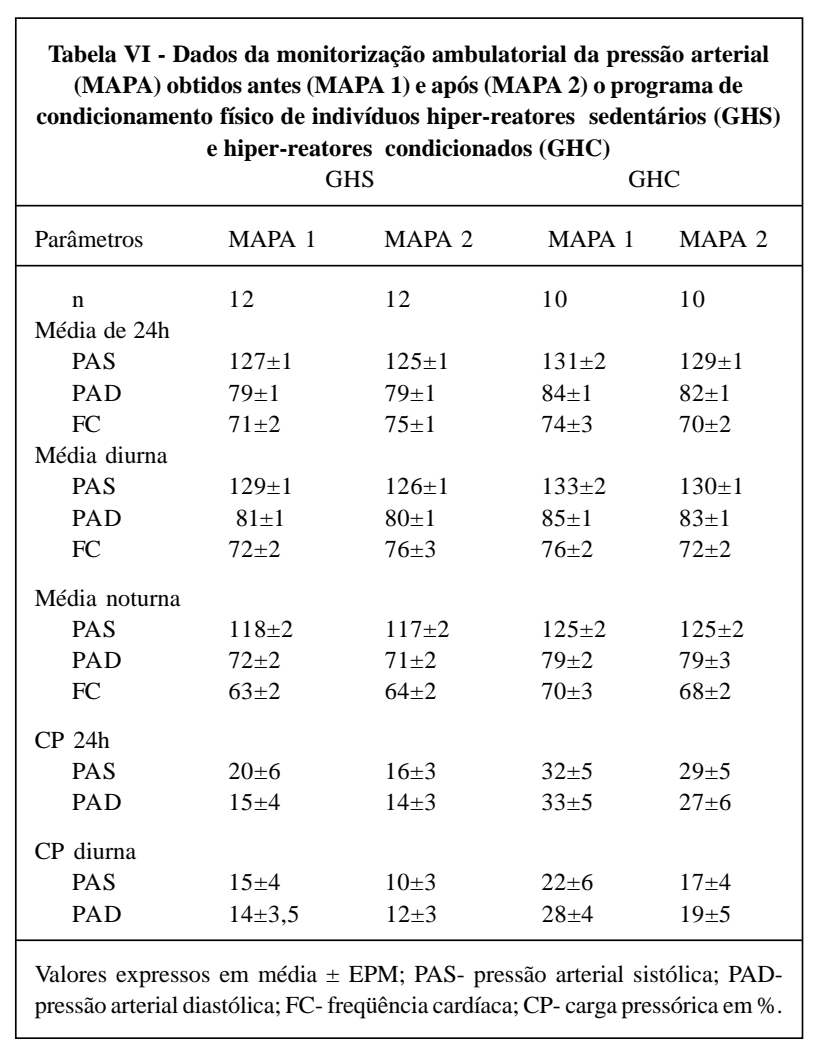

Foram analisados além das médias de PAS e PAD em $24 \mathrm{~h}$ e em subperíodos, a FC de $24 \mathrm{~h}$ e a variabilidade pressórica.

O relatório final contendo todos os dados da monitorização foi obtido através de um microcomputador IBM-486 munido de impressora.

O GHC foi submetido a programa de exercícios físicos

\begin{tabular}{|c|c|c|c|c|}
\hline \multirow{2}{*}{\multicolumn{5}{|c|}{$\begin{array}{c}\text { Tabela VII - Variabilidade da pressão arterial obtida da monitorização } \\
\text { ambulatorial da pressão arterial (MAPA) entre os grupos hiper-reatores } \\
\text { sedentários (GHS) e hiper-reatores condicionados (GHC) antes (teste 1) e } \\
\text { após (teste 2) o programa de condicionamento físico } \\
\text { GHS }\end{array}$}} \\
\hline & & & & \\
\hline Parâmetros & MAPA 1 & MAPA 2 & MAPA 1 & MAPA 2 \\
\hline $\mathrm{n}$ & 12 & 12 & 10 & 10 \\
\hline \multicolumn{5}{|l|}{ DP de $24 \mathrm{~h}$} \\
\hline PAS & $10,6 \pm 0,3$ & $11,0 \pm 0,6$ & $12,0 \pm 0,6$ & $10,3 \pm 0,8$ \\
\hline PAD & $9,2 \pm 0,4$ & $9,5 \pm 0,6$ & $10,3 \pm 0,6$ & $9,4 \pm 0,8$ \\
\hline \multicolumn{5}{|l|}{ DP diurna } \\
\hline PAS & $9,4 \pm 0,3$ & $10,1 \pm 0,7$ & $10,0 \pm 0,7$ & $10,0 \pm 0,8$ \\
\hline PAD & $7,9 \pm 0,3$ & $8,5 \pm 0,6$ & $8,8 \pm 0,8$ & $8,5 \pm 0,9$ \\
\hline \multicolumn{5}{|l|}{ DP noturna } \\
\hline PAS & $9,0 \pm 1,0$ & $8,8 \pm 1,1$ & $3,4 \pm 1,0$ & $10,0 \pm 0,8$ \\
\hline PAD & $8,7 \pm 0,8$ & $8,4 \pm 1,0$ & $1,5 \pm 0,7$ & $9,8 \pm 0,7$ \\
\hline \multicolumn{5}{|l|}{$\mathrm{CV}$ de $24 \mathrm{~h}$} \\
\hline PAS & $8,4 \pm 0,3$ & $8,7 \pm 0,4$ & $9,1 \pm 0,4$ & $8,0 \pm 0,6$ \\
\hline PAD & $11,5 \pm 0,5$ & $12,0 \pm 0,7$ & $12,2 \pm 0,6$ & $11,3 \pm 0,9$ \\
\hline \multicolumn{5}{|l|}{ CV diurna } \\
\hline PAS & $7,2 \pm 0,3$ & $8,0 \pm 0,6$ & $7,5 \pm 0,5$ & $7,7 \pm 0,6$ \\
\hline PAD & $9,7 \pm 0,4$ & $10,6 \pm 0,8$ & $10,3 \pm 0,9$ & $10,3 \pm 1,1$ \\
\hline \multicolumn{5}{|l|}{$\mathrm{CV}$ noturna } \\
\hline PAS & $7,6 \pm 0,8$ & $7,4 \pm 0,8$ & $10,6 \pm 0,7$ & $8,0 \pm 0,7^{*}$ \\
\hline PAD & $11,9 \pm 1,0$ & $11,6 \pm 1,3$ & $14,4 \pm 0,6$ & $12,5 \pm 1,0$ \\
\hline
\end{tabular}

Valores expressos como média \pm EPM; DP- desvio padrão; $\mathrm{CV}$ - coeficiente de variação; *p<0,05 (GHC - MAPA 1 vs MAPA 2) - teste t Student. 
aeróbicos e após quatro meses, todos os indivíduos foram reavaliados através de TE e MAPA, objetivando análise de parâmetros ergométricos e cardiovasculares.

O comparecimento às sessões foi superior a $80 \%$.

A comparação das médias entre os grupos foi feita aplicando-se o teste de $t$ de Student para amostras pareadas e independentes. Para a comparação entre as várias médias obtidas, realizamos a análise de variância (ANOVA), seguido de teste de Tukey para comparação dos diferentes pares de média, procurando identificar a significância existente entre elas. Os resultados foram expressos em média \pm erro padrão da média. Foram considerados estatisticamente significantes os valores de probabilidades $\mathrm{P}<0,05^{13}$.

Para a correlação entre duas variáveis quantitativas utilizamos o coeficiente de correlação simples de Pearson. Para medida de dispersão absoluta, utilizamos o desvio padrão e para medida de dispersão relativa, usamos o coeficiente de variação. Todos os cálculos estatísticos foram realizados utilizando-se o programa GB-STAT V 500, 1993.

\section{Resultados}

$\mathrm{Na}$ tabela I estão representadas as características antropométricas e etárias iniciais de ambos os grupos. A comparação entre as cifras tensionais e a FC no TE obtidas
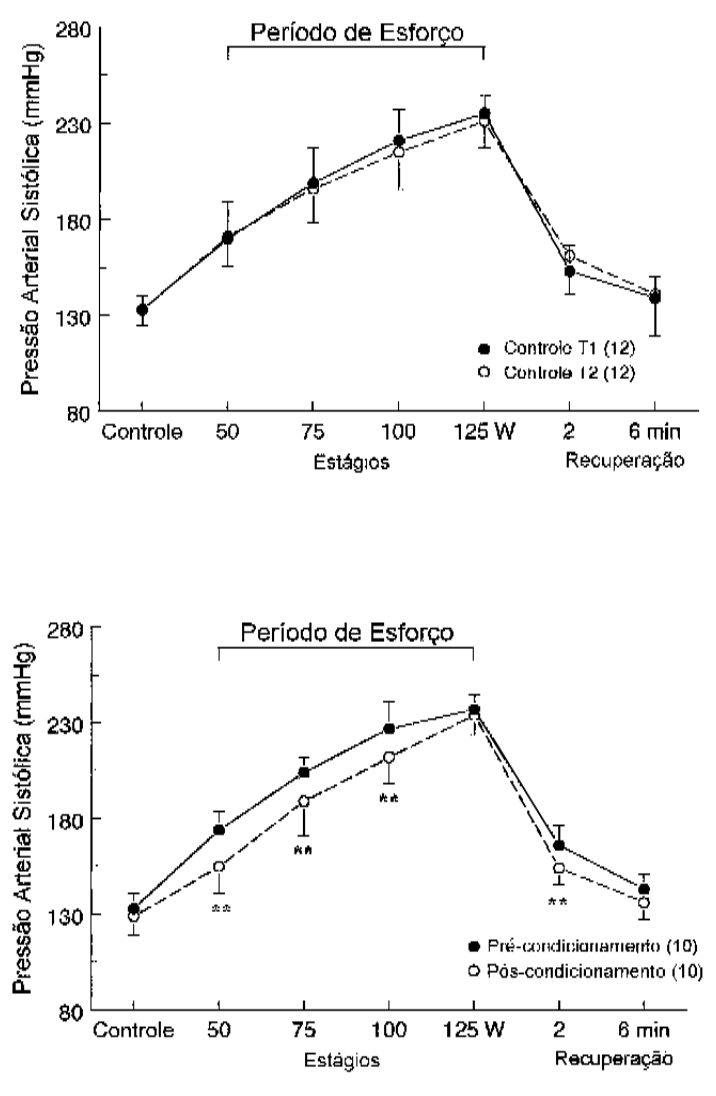

Fig. 1 - Comportamento dos valores pressóricos sistólicos frente ao teste ergométrico entre o grupo controle e o grupo submetido a programa de condicionamento físico. Os valores indicam a média $\pm \mathrm{EPM}$. $* \mathrm{p}<0,05 \mathrm{e} * \mathrm{p}<0,01$.

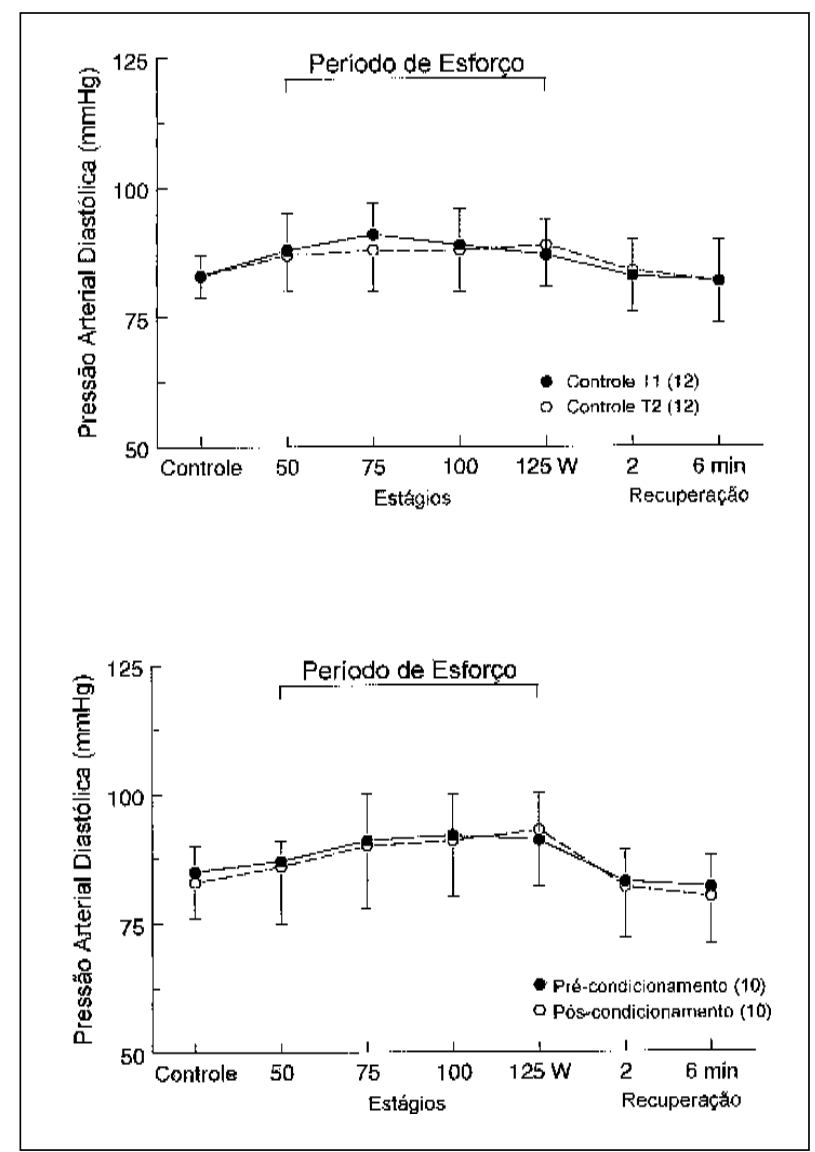

Fig. 2 - Comportamento dos valores pressóricos diastólicos frente ao teste ergométrico entre o grupo controle e o grupo submetido a programa de condicionamento físico. Os valores indicam a média \pm EPM.

antes do condicionamento (teste 1) e após (teste 2), mostrou tendência à redução dos níveis pressóricos em GHC, não havendo, entretanto, significância estatística (tab. II).

Na comparação entre os TE realizados antes e após o programa de exercícios, observamos redução significativa dos níveis tensionais sistólicos em cargas sub-máximas de trabalho e durante $\mathrm{o} 2^{\circ}$ minuto de recuperação em GHC (fig. 1). Os valores obtidos de PAS nos correspondentes estágios foram semelhantes quando os testes 1 e 2 dos indivíduos de GHS foram comparados.

Na comparação dos valores de PAD durante o esforço ergométrico nos testes 1 e 2, não foram observadas modificações significativas, quando ambos os grupos foram analisados (fig. 2).

Em relação à FC, observamos redução significativa dos valores em cargas submáximas de trabalho e no período de recuperação nos indivíduos condicionados. No GHS não foram verificadas diferenças significantes de FC durante as diversas fases do TE (fig. 3).

A análise da duração do teste e trabalho total estão representadas na tabela III. Podemos observar que houve um aumento significativo na duração do teste $(p<0,05)$ e aumento não significativo do trabalho total no grupo condicionado no teste 2 .

A análise do duplo produto (PAS x FC) obtido durante 

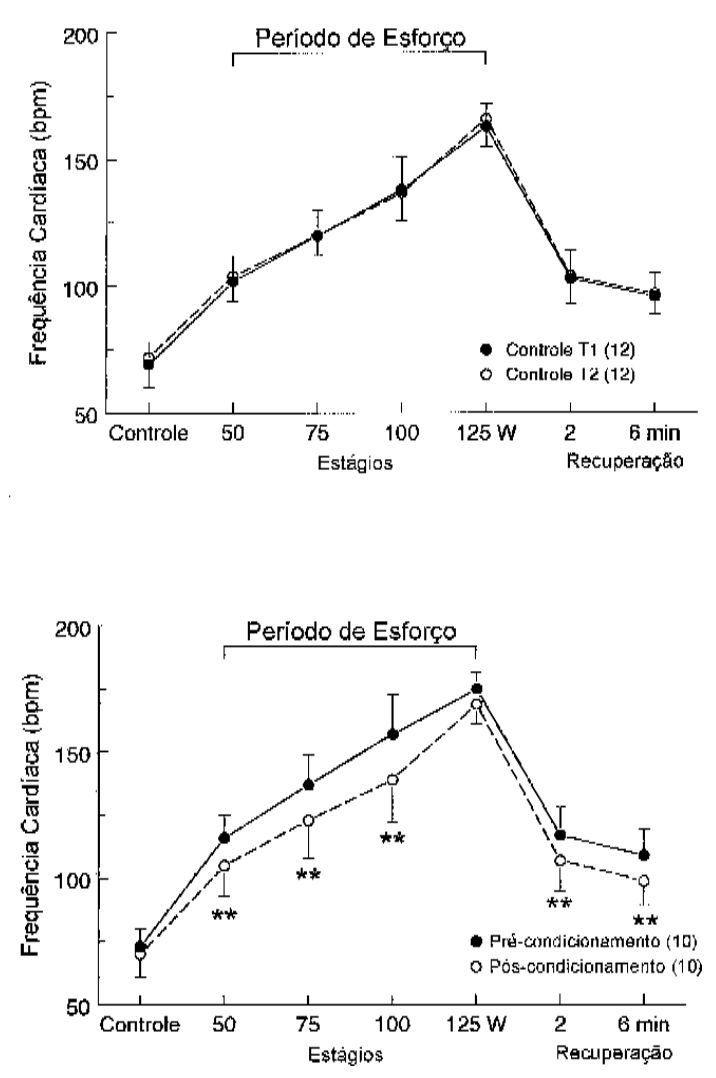

Fig. 3-Comportamento dos valores de freqüência cardíaca frente ao teste ergométrico entre o grupo controle e o grupo submetido a programa de condicionamento físico. Os valores indicam a média \pm EPM. $* \mathrm{p}<0,05 \mathrm{e}^{* *} \mathrm{p}<0,01$

o TE no teste 2 mostra redução significativa no GHC durante as cargas de trabalho, não observada no GHS (tab. IV e V).

A comparação dos dados obtidos pela MAPA antes e depois do programa de condicionamento físico mostra ausência de significância estatística na PAS e PAD em ambos os grupos (tab. VI e VII). Os valores de FC também foram semelhantes para os grupos estudados.

\section{Discussão}

Quando os resultados do condicionamento físico em hiper-reatores foram analisados, observamos a ocorrência de adaptações crônicas, resultantes da ação do condicionamento físico sobre o sistema cardiovascular. Essas modificações são caracterizadas por redução da PAS e FC em cargas sub-máximas de trabalho, redução de duplo produto e aumento da duração do exercício, bem como maiores valores de trabalho total. Todavia, não observamos redução significativa nos valores de PAS e PAD e de FC nas condições de repouso.

Os efeitos que um programa de exercícios exerce sobre os níveis de PA em repouso têm sido motivo de controvérsias. Assim, em vários estudos longitudinais, não foram encontradas diferenças significativas da PA nesta condição ${ }^{14-20,}$, fato que também verificamos no nosso estudo. Outros pesqui- sadores, entretanto ${ }^{21-24}$ têm documentado pequena, mas significativa redução dos valores pressóricos em repouso. $\mathrm{Na}$ interpretação desses resultados, os aspectos metodológicos exercem grande influência. Vários fatores poderiam estar influenciando, como: ausência de grupo controle, critérios de randomização, determinaçãonão-cega dos níveis tensionais e possibilidade de ocorrerem efeitos por co-intervenção.

Analisando o comportamento dos valores sistólicos frente ao TE, observamos que os indivíduos condicionados apresentaram redução dos níveis sistólicos em todas as cargas sub-máximas de trabalho e no $2^{\circ}$ minuto do período de recuperação. Redução significativa dos níveis sistólicos também foi observada em carga sub-máxima de trabalho por outros autores ${ }^{6,23-25}$ e pode ser conseqüência de uma atenuação da atividade simpática secundária ao treinamento e diminuição dos níveis de noradrenalina plasmática ${ }^{26-28}$. A análise dos valores de PAD mostrou comportamento fisiológico entre os grupos, não só durante o decorrer do esforço, como no período de recuperação ${ }^{10}$. Os valores de FC em repouso foram semelhantes para ambos os grupos.

Pelos nossos resultados, na condição de repouso não observamos adaptações crônicas resultantes da ação do condicionamento físico sobre o sistema cardiovascular. Essas modificações segundo Frick e $\mathrm{col}^{29}$ são caracterizadas por redução de FC de repouso relacionada, possivelmente, por aumento do tônus parassimpático.

Existem evidências de que o mecanismo desta bradicardia no homem se deva principalmente, a uma diminuição da FC intrínseca ${ }^{30}$ e não à modificação do tônus parassimpático.

Os dados de Maciel e col ${ }^{31}$, avaliando o tônus vagal sobre o nódulo sinusal em indivíduos sedentários, antes e após o programa de exercícios físicos, mostram a contribuição parassimpática na bradicardia exercício induzida. Efeitos bradicardizantes secundários ao condicionamento físico não foram observados por outros autores ${ }^{32-35}$.

Os valores de FC obtidos em nosso estudo, durante o exercício submáximo, foram significativamente menores em todas as cargas de trabalho e no período de recuperação no GHC quando comparados ao GHS, evidenciando provável efeito cronotrópico negativo secundário ao treinamento, concordando com os trabalhos de Saltin e col ${ }^{17}$, Clausen ${ }^{36} \mathrm{e}$ Maciel e col ${ }^{31}$ que observaram, em indivíduos sedentários treinados, discreta mas significativa redução de FC esforço induzida, provavelmente, por diminuição da atividade simpática sobre o coração.

Os valores de duplo produto de GHC foram significativamente menores, quando comparados ao GHS durante o exercício submáximo, e no período de recuperação. Observamos também aumento significativo na duração do esforço e maiores valores de trabalho total, evidenciando, assim, um maior desempenho físico nos indivíduos treinados ${ }^{37}$.

Na impossibilidade de medida do consumo direto de $\mathrm{O}_{2}$ nesses indivíduos e considerando que as equações de determinação indireta do consumo máximo de oxigênio $\left(\mathrm{VO}_{2}\right.$ max) apresentam um erro de predição de $15 \%{ }^{38} \mathrm{e}$, portanto, não recomendável a sua utilização em pesquisa, não demos 
ênfase a esta variável. Vários trabalhos na literatura também não utilizam o $\mathrm{VO}_{2}$ max como parâmetro de melhora de desempenho cardiorrespiratório, quando indivíduos normotensos e hipertensos são submetidos a programa de condicionamento físico ${ }^{20,39-43}$.

Quando analisamos os parâmetros da MAPA, não observamos redução dos valores de PAS e PAD durante as 24h e em subperíodos, quando as médias de GHC foram comparadas com as de GHS.

Gilders e $\mathrm{col}^{44}$, estudando 21 pacientes adultos normotensos e hipertensos ( $43 \pm 4$ anos), sendo 10 submetidos a programa de condicionamento físico por 16 semanas, não observaram alterações na pressão ambulatorial de $24 \mathrm{~h}$ e na pressão noturna nesses indivíduos. Observaram aumento de $\mathrm{VO}_{2}$ max de aproximadamente $14 \%$ e redução de $\mathrm{FC}$ em repouso. Segundo esses autores, o $\mathrm{VO}_{2}$ max e os valores de pressão ambulatorial são variáveis independentes, ou seja, o treinamento aeróbico em indivíduos normotensos não implica redução dos níveis tensionais.

Em trabalho recente, Wijnen e col $^{35}$ avaliaram 19 indivíduos sedentários, do sexo masculino com idade entre $22 \mathrm{e}$ 44 anos, normotensos e hipertensos leves, submetidos a programa de condicionamento físico em cicloergômetro por um período de seis semanas, com frequiência de três vezes por semana, duração de $45 \mathrm{~min}$ por sessão e com intensidade de esforço correspondente a $75 \%$ do $\mathrm{VO}_{2}$ max. Os autores observaram aumento do consumo máximo de oxigênio e redução da FC de repouso; entretanto, quando os valores de MAPA foram comparados, não foram observadas variações significativas dos níveis pressóricos, antes e após o programa de exercícios físicos aeróbicos. Nossos resultados vão de encontro aos obtidos por esse estudo; entretanto, devendo ser levado em consideração que apesar de obter aumento do $\mathrm{VO}_{2}$ max, Wijnen e $\mathrm{col}^{35}$ realizaram o treinamento apenas por seis semanas.

Em contrapartida, Van Hoof e $\mathrm{col}^{25}$ estudaram o efeito do condicionamento físico na PA de repouso e durante a MAPA de 24h, em 26 indivíduos sedentários, do sexo masculino, com média de $38 \pm 10$ anos. Esses indivíduos foram submetidos a um programa de exercícios de 48 sessões, e com uma frequiência de três vezes por semana. Observaram redução apenas dos níveis diastólicos em repouso. A PA, quando medida durante o TE submáximo, mostrou redução dos níveis sistólicos para cargas submáximas de trabalho. Quando medida durante 24h, o treinamento determinou redução dos níveis pressóricos diastólicos durante o dia, de 89 para $84 \mathrm{mmHg}$. A pequena alteração observada na PAS não foi significante. Não foram registradas diferenças significativas nos níveis sistólicos e diastólicos durante o período noturno. Observaram também redução dos valores de FC tanto no período diurno como no período noturno. Estudando a variabilidade pressórica, encontraram valores significativos apenas para PAD diurna, de $8 \pm 3 \%$ para $7 \pm \%$ (controle vs treinados), não encontrando influência do treinamento físico sobre o coeficiente de variação da PAS diurna ou noturna e nem na diastólica noturna.

Estudamos a variabilidade de PA comparada entre
GHS e GHC e não observamos (exceto para a PAS noturna), diferenças, estatisticamente, significantes para os coeficientes de variação.

Quando os valores de carga pressórica foram comparados, observamos resultados semelhantes antes e após o período de exercícios. Estes resultados vêm ao encontro dos obtidos por Marceau e $\mathrm{col}^{45}$ que observaram redução de carga pressórica secundária ao treinamento de baixa e moderada intensidade. Neste estudo, o treinamento foi realizado em indivíduos hipertensos, que são mais sensíveis à redução dos níveis tensionais (World Hypertension League $)^{7}$ secundários aos exercícios, ou seja, com uma população diferente da do nosso estudo.

A análise do perfil das curvas de PAS, PAD e de FC obtidas de $2 \mathrm{em} 2 \mathrm{~h}$ durante as $24 \mathrm{~h}$ evidencia semelhança entre o obtido antes e após o programa de condicionamento físico, não existindo uma queda mantida e uniforme dos níveis tensionais durante as $24 \mathrm{~h}$ e/ou em subperíodos. Resultados semelhantes foram obtidos por Van Hoof e $\mathrm{col}^{25}$.

Em relação ao duplo produto da MAPA, não observamos modificações significativas quando GHS e GHC foram comparados entre si. Estes resultados estão de acordo com os obtidos por Gilders e $\mathrm{col}^{44}$, que estudaram através da MAPA indivíduos normotensos e hipertensos submetidos a programa de exercícios físicos aeróbicos.

$\mathrm{Na}$ análise dos mecanismos envolvidos nas variações da PA após o condicionamento físico, Jennings e $\mathrm{col}^{46} \mathrm{e}$ Meredith e $\mathrm{col}^{47,48}$ atribuem ser o efeito hipotensor secundário à redução da resistência vascular periférica. Redução da RVP também foi observada no estudo de Martin e $\mathrm{col}^{49}$, embora não houvesse redução dos níveis pressóricos. Em outros estudos, Gilders e col, Wolfe e col e Cononie e col ${ }^{44,50,51}$, os níveis pressóricos e outras variáveis hemodinâmicas não foram influenciadas pelo condicionamento físico.

Fatores humorais também estão envolvidos, tais como redução dos níveis plasmáticos de noradrenalina secundária à diminuição da atividade simpática ${ }^{3}$. Outro fator que poderia contribuir para a atenuação da resposta simpática pós-condicionamento físico seria a diminuição da sensibilidade beta-adrenérgica.

Os efeitos do condicionamento sobre o controle cronotrópico pelos barorreceptores são controversos ${ }^{52,53}$. Estudos sobre a reatividade vascular têm mostrado menor reatividade ao estresse cardiovascular cotidiano, podendo este fator contribuir para o efeito hipotensor do treinamento aeróbico ${ }^{54}$.

Atribui-se também o aumento da volemia e conseqüente aumento do volume de ejeção cardíaca como um dos fatores responsáveis pela diminuição da FC, via efeito FrankStarling, após condicionamento físico ${ }^{55}$.

Concluímos com base nos nossos resultados que ao implementarmos um programa de condicionamento físico aeróbico em indivíduos apresentando elevação exagerada da PAS ao exercício físico dinâmico, os níveis tensionais e os valores de FC, quando estudados pela medida convencional e pela MAPA, não apresentaram alterações estatisticamente significantes, questionando, assim, os benefícios do 
exercício físico como medida isolada na redução das cifras pressóricas de $24 \mathrm{~h}$ e em subperíodos. Entretanto, a atividade inotrópica e cronotrópica, em cargas submáximas de trabalho durante a realização do TE, apresentaram redução significativa, secundária possivelmente a uma atenuação da atividade simpática motivada pelo treinamento. Portanto, investigações adicionais serão necessárias para esclarecimentos de como os fundamentos básicos da prescrição de exercícios físicos (intensidade, duração e frequiência) podem contribuir para uma redução efetiva da PA, visando uma maior contribuição da atividade física na prevenção primária daHAS.

\section{Referências}

1. Tipton CM - Exercise training and hypertension. Exerc Sport Sci Rev 1984; 12: 245-306.

2. Fuchs FD, Moreira WD, Ribeiro JP - Eficácia anti-hipertensiva do condicionamento físico aeróbico. Uma análise crítica das evidências experimentais. Arq Bras Cardiol 1993; 61: 187-90.

3. Arroll B, Beaglehole R - Does physical activity lower blood pressure: a critical review of the clinical trials. J Clin Epidemiol 1992; 45: 437-9.

4. Hellerstein HK, Hornstein TR - Assessing and preperring a patient for return to a meaningful and productive life. J Rehab 1972; 32: 602.

5. Holmann W, Rest R, Liesen $\mathrm{H}$ - The importance of sport and physical training in preventive cardiology. J Sports Med Phys Fitness 1980; 20: 5.

6. Lima EG, Nascimento LMF, Busatto VCW, Rocha RC - Avaliação cardiorrespiratória em indivíduos sedentários durante programa de condicionamento físico. Arq Bras Cardiol 1983; 40: 11-3.

7. Physical exercise in the management of hypertension: a consensus statement by the World Hypertension League. J Hypertens 1991; 9: 283-7.

8. Arakawa K - Hypertension and exercise. Clin Exp Hypertens 1993; 15: 1171-9.

9. Couto HA - Temas de Saúde Ocupacional. Belo Horizonte: Cultura, 1987.

10. Chaitman B - Exercise stress testing. In: Braunwald E-Heart Disease. Philadelphia: WB Saunders, 1992: 170.

11. American College of Sports Medicine position stand - Physical activity, physical fitness and hypertension. Med Sci Sports Exerc 1993; 25: i-x.

12. II Consenso Brasileiro para o Uso da Monitorização Ambulatorial da Pressão Arterial. HiperATIVO 1996; 3: 237-49.

13. Dixon WJ, Massey FJ Jr - Introduction to statistical analysis. New York: McGraw Hill, 1969.

14. Montoye WE, Oldham PD - Factors influencing arterial blood pressure in the general population. Clin Sci 1958; 17: 409-44.

15. Frick MH, Konttinen A, Sarajas HSS - Effects of physical training on circulation at rest and during exercise. Am J Cardiol 1963; 12: 142.

16. Tabakin BSS, Hanson JS, Levy AM - Effects of physical training on the cardiovascular and respiratory response to graded upright exercise in distance runners. $\mathrm{Br}$ Heart J 1965; 27: 205 .

17. Saltin B, Blomqvist G, Mitchell JH et al - Response to exercise after bed rest and after training. Circulation 1968; 38: 1.

18. Ekblom B, Astrand P, Saltin B et al - Effect of training on circulatory response to exercise. J Appl Physiol 1968; 24: 518-28.

19. Hanson J, Tabakin B, Levy A et al - Long-term physical training and cardiovascular dynamics in middle-aged men. Circulation 1968; 38: 783-99.

20. Arida RM, Naffah-Mazzacoratti MG, Soares J et al - Effect of an aerobic exercise program on blood pressure and cathecolamines in normotensive and hypertensive subjects. Brazilian Med Biol Res 1996; 29: 633-7.

21. Cogswell RC, Henderson CR, Berryman CH et al - Some observations on the effects of training on pulse rate, blood pressure and endurance in humans using the step test (Harvard), treadmill and electrodynamic brake bicycle ergometer. Am J Physiol 1946; 146: 422.

22. Barry AJ, Daly JW, PruettEDR et al - The effects of physical conditioning on older individuals. I Work capacity, circulatory - respiratory function and work electrocardiogram. J Gerontol 1966; 21: 182.

23. Roman O, Camuzzi Al, Villalon E et al - Physical training program in arterial hypertension. A long-term prospective follow-up. Cardiology 1981; 67: 230-43.

24. Hagberg JM, Goldring D, Ehsani AA et al - Effect of exercise on the blood pressure and hemodynamic features of hypertensive adolescents. Am J Cardiol 1983; 52: $763-8$

25. Van Hoof R, Hespel P. Fagard R et al - Effect of endurance training on blood pressure at rest, during exercise an during 24 hours in sedentary men. Am J Cardiol 1989; 63: 945-9.

26. Kiyonaga A, Arakawa K, Tanaka H et al - Blood pressure and hormonal responses to aerobic exercise. Hypertension 1985; 7: 125-31.

27. Urata H, Tanabe Y, Kiyonaga A et al - Antihypertensive and volume- depleting effects of mild exercise on essential hypertension. Hypertension 1987; 9: 245-52.

28. Duncan JJ, Farr JE, Upton SJ et al - The effects of aerobic exercise on plasma cathecolamines and blood pressure in patients with mild essential hypertension. JAMA 1985; 254: 2609-13.
29. Frick MH, Elovaínio RO, Somer T - The mechanism of bradicardia evoked by physical training. Cardiologia 1967; 51: 46.

30. Katona PG, McLean M, Dighton PH et al - Sympathetic and parasympathetic cardiac control in athletes and nonathletes at rest. J Appl Physiol 1982; 52: 1652.

31. Maciel BC, Gallo Jr L, Marin Neto JA et al - Parasympathetic contribution to bradycardia induced by endurance training in man. Cardiovasc Res 1985; 19: 642.

32. Martin JE, Dubbut PM, Cushman WC - Controlled trial of aerobic exercise in hypertension. Circulation 1990; 81: 1560-7.

33. Blumenthal JA, Siegel WC, Appelbaum M - Failure of exercise to reduce blood pressure in patients with mild hypertension. JAMA 1991; 266: 2098-104.

34. Grassi G, Seravalle G, Calhoun AD, Mancia G-Physical training and baroreceptor control of sympathetic nerve activity in humans. Hypertension 1994; 23 294-301.

35. Wijnen JAG, Kool MJF, Van Boak MA et al - Effect of exercise training on ambulatory blood pressure. Intern J Sports Medic 1994; 15: 10-5.

36. Clausen JP - Effects of physical training on cardiovascular adjustments to exercise in man. Physiol Rev 1977; 779-815.

37. Johnson WP, Grover JA-Hemodynamic and metabolic effects of physical training in four patients with essential hypertension. Can Med Assoc J 1967; 96: 842-6.

38. Astrand PO, Rhyming I- Normogram for calculation of aerobics capacity (physical fitness) from pulse during submaximal work. J Appl Physiol 1954; 7: 218-21.

39. Erikssen J, Jerwell J, Forfang K-Blood pressure response to bicycle exercise testing in apparently healthy middle-aged men. Cardiology 1980; 66: 56-64.

40. Wilcox RG, Bennett T, Brown AM, Mac Donald IA - Is exercise good for high blood pressure? Clin Res 1982; 285: 767-9.

41. Cade R, Mars D, Wagemaker Het al-Effect of aerobic exercise training on patients with systemic arterial hypertensionn. Am J Med 1984; 77: 785-90.

42. Kiyonaga A, Arakawa K, Tanaka H, Shindo M - Blood pressure and hormonal responses to aerobic exercise. Hypertension 1985; 7: 125-31.

43. Nelson L, Esler MD, Jennings GL, Korner PI-Effectt of changing levels of physical activity on blood pressure and haemodynamic in essential hypertension. Lancet 1986; 473-7.

44. Gilders RM, Voner C, Dudley GA - Endurance training and blood pressure in normotensive and hypertensive adults. Med Sci Sports Exerc 1989; 21: 629-36.

45. Marceau M, Kouamé N,Lacourcière Y,Cléroux J-Effects of different training intensities on 24-hour blood pressure in hypertensive subjects. Circulation 1993; 88: 2803-11.

46. Jennings $\mathrm{G}, \mathrm{Nel}$ son $\mathrm{L}, \mathrm{Nestel} \mathrm{P}$ et al - The effects of changes in physical activity on major cardiovascular risk factors, hemodynamics, sympathetic function and glucose utilization in man: a controlled study of four levels of activity. Circulation 1966; 73: 30-40

47. Meredith IT, Jennings GL, Esler MD - Time-course of the antihypertensive and autonomic effects of regular endurance exercise in human subjects. J Hypertens 1990; 8: 859-66.

48. Meredith IT, Friberg P, Jennings GL - Exercise training lowers resting renal but not cardiac sympathetic activity in humans. Hypertension 1991; 18: 575-82.

49. Martin WD, Montgomery J, Snell PG - Cardiovascular adaptations to intense swim training in sedentary middle-aged men and women. Circulation 1987; 75 : 323-30.

50. Wolfe LA, Cunningham DA, Rechnitzer PA-Effects of endurance training on left ventricular dimensions in healthy men. J Appl Physiol 1979; 47: 207-12.

51. Cononie CC, Graves JE, Pollock ML et al - Effect of exercise training on blood pressure in 70 to 79-yr-old men and women. Med Sci Sports Exerc 1991; 23:50511.

52. Smith ML, Graitzer HM, Hudson DH, Raven PB - Baroreflex function in endurance and static exercise-trained men. J Appl Physiol 1988; 64: 585-91.

53. Shi X, Andressen JM, Potts JT et al - Aortic baroreflex control of heart rate during hypertensive stimuli: effect of fitness. J Appl Physiol 1993; 74: 1555-62.

54. Clayton RP - Stress reactivity: hemodynamic adjustments in trained and untrained humans. Med Sci Sports Exerc 1991; 25: 873-81.

55. Elias AN, Domurat ES, Pandian MR et al - Concentrations of volume-regulatory hormones after exercise in physically conditioned males. J Med 1991; 22: 55-67. 